\title{
Estrategias de afrontamiento en adultos mayores en función de variables sociodemográficas
}

\section{Coping strategies in older adults as a function of sociodemographic variables}

\author{
Teresa Mayordomo Rodríguez, Alicia Sales Galán, Encarnación Satorres Pons \\ y Clara Blasco Igual
}

Universidad de Valencia, España

Disponible online 31 de diciembre de 2015

\begin{abstract}
Para conseguir la adaptación a los cambios que se producen en el envejecimiento se ponen en marcha una serie de estrategias de afrontamiento. En este estudio, 275 adultos mayores, que acuden a un centro de mayores a realizar actividades, respondieron a una serie de datos sociodemográficos y al Cuestionario de Afrontamiento del Estrés (CAE) mediante una entrevista semiestructurada. El objetivo es analizar el uso de las estrategias de afrontamiento en función de diversas variables sociodemográficas, mediante el uso de correlaciones, pruebas t y ANOVAs. Los resultados mostraron una relación positiva y significativa entre la edad y la religión, y se observaron diferencias en función del género, del estado civil, del nivel de estudios y en función del nivel de ingresos. Como conclusión puede decirse que a medida que aumenta la edad la estrategia religión es más utilizada; las mujeres hacen mayor uso de la evitación, la búsqueda de apoyo social y la religión; los solteros utilizan más la búsqueda de apoyo social y la reevaluación positiva, mientras que los viudos hacen mayor uso de la religión; las personas con estudios inferiores utilizan más la autofocalización negativa y la evitación y las personas con mayores ingresos utilizan más la estrategia focalizado en solución de problemas, y aquellos con ingresos inferiores hacen mayor uso de la estrategia religión.
\end{abstract}

Palabras Clave: Adultos Mayores; Adaptación; Estrategias de Afrontamiento; Variables Sociodemográficas.

Coping strategies are used to adapt to the changes that occur during aging. This study investigated 275 older adults who attended a senior citizen's centre. They provided sociodemographic data and answered the Stress Coping Questionnaire (SCQ) in a semistructured interview. The aim was to analyse the use of coping strategies based in several sociodemographic variables, using correlations, $t$ tests, and ANOVAs. The results showed a positive and significant association between age and religion, and differences were found in gender, marital status, educational attainment, and income level. In conclusion, as age increases, religion is most commonly used coping strategy; women make greater use of avoidance, seek social support, and use religion; singles mainly seek social support and positive reappraisal; widowers make greater use of religion; people with a lower level of education use more negative self-targeting and avoidance; people with higher incomes mainly use strategies centred on solving problems; and those with low incomes make greater use of religion.

Key Words: Older Adults; Adaptation; Coping Strategies; Sociodemographic Variables.

Correspondencia: Teresa Mayordomo Rodríguez. Departamento de Psicología Evolutiva y de la Educación, Facultad de Psicología, Universidad de Valencia. Avda Blasco Ibáñez, 21. 46010-Valencia. Tlf. 96383844. E-mail: teresamayordomor@gmail.com. E-mails de los co-autores: Alicia Sales Galán: Alicia.sales@uv.es, Encarnación Satorres Pons: encarsatorres@gmail.com, Clara Blasco: M.Clara.Blasco@uv.es. 
Durante el envejecimiento, existe una disminución de los recursos tanto materiales como personales asociados a la edad, que dificultan el ajuste ante situaciones desventajosas como la pérdida de salud, pérdida de seres queridos, el aumento de la dependencia,etc., (Martin, Kliegel, Rott, Poon y Johnson, 2008), que interfieren en la vida cotidiana del adulto mayor. Independientemente de estas pérdidas, el adulto mayor sigue manteniendo recursos disponibles para modificar aquellas situaciones desadaptativas y conseguir un ajuste óptimo (Freund y Riediger 2001); además, mantiene la capacidad para la adquisición de nuevos recursos de afrontamiento que pueden reducir el impacto negativo de los eventos estresantes (Taylor y Stanton 2007). La puesta en marcha de éstas estrategias de afrontamiento de una forma efectiva facilitaría un envejecimiento satisfactorio.

El afrontamiento, ha sido definido como aquellos esfuerzos cognitivos y conductuales constantemente cambiantes que se desarrollan para manejar las situaciones específicas externas y/o internas que son evaluadas como excedentes o desbordantes de los recursos del individuo (Lazarus y Folkman, 1984). Desde esta perspectiva, existen dos tipos de afrontamiento, uno centrado en el problema, que tiene como objeto manejar o alterar el problema que está causando el malestar; y otro centrado en la emoción, que implica métodos dirigidos a regular la respuesta emocional ante el problema.

Por lo que respecta al funcionamiento de las estrategias de estos dos tipos de afrontamiento en la última etapa del ciclo vital, Folkman, Lazarus, Pimley y Novacek (1987) mostraron que las personas mayores tienen mayor capacidad para tomar distancia con respecto a las situaciones estresantes y revaluarlas positivamente, además se ha comprobado que los adultos mayores pasan de un estilo centrado en el problema a uno, en mayor medida, centrado en la emoción (Carver y Connor-Smith 2010); tal y como señalaba Labouvie-Vief (1999) las personas mayores saben identificar mejor sus emociones que los jóvenes y elaboran mejores y más apropiadas estrategias para actuar eficazmente en las situaciones conflictivas.

En relación a la edad, Trouillet, Gana, Lourel y Fort (2009) observaron cómo ésta predice cambios en los recursos de afrontamiento, incluyendo la disminución del apoyo social y el aumento de los niveles de estrés percibido; estas modificaciones implican un mayor uso del afrontamiento centrado en la emoción, de este modo, los intentos de controlar la tensión interna, se afrontarían evitando situaciones de estrés o reevaluando positivamente situaciones aversivas.

Por lo que respecta al género, Rose y Rudolph (2006) indican que incluso en etapas tempranas del desarrollo, hombres y mujeres no se enfrentan del mismo modo al estrés. Según Tamres, Janicki y Helgeson (2002), los hombres generalmente se enfrentan de forma directa al problema o lo niegan, mientras que las mujeres responden de un modo más emocional a las situaciones estresantes y se apoyan más en otros, ya sean amigos o familiares. Del mismo modo, Kirchner, Forns, Muñoz y Pereda (2008) postulan que mientras los hombres tienden a regular sus estados emocionales mediante el uso de más evitación conductual que cognitiva, las mujeres utilizan esas dos tácticas de evitación con una muy similar frecuencia.

En cuanto al resto de variables sociodemográficas que pueden influir en el afrontamiento, autores como Navarro y Bueno (2005), estudiaron el papel que juegan éstas en las estrategias de afrontamiento para población mayor señalando diferencias en función del nivel económico. Por lo que respecta al nivel de estudios y según Birren (1994), la principal influencia que tiene el nivel de escolaridad es la capacidad para maximizar y conservar los propios recursos que se ven implicados en la toma de decisiones de la vida cotidiana. Tanto la educación como los aprendizajes adquiridos a lo largo de la vida, pueden proporcionar claves necesarias para la adaptación en el envejecimiento. Además, las personas con alto nivel de escolaridad son más efectivas en su vida cotidiana ya que buscan información, la discuten, etc. Por lo que respecta al nivel económico, se indica que las personas mayores que no tengan suficientes ingresos garantizados pueden incidir de forma negativa sobre su salud e independencia, considerándose de manera subjetiva como menos eficaces a la hora de enfrentarse a determinadas situaciones. Algunos autores (Holahan y Moos, 1987), que vinculan el afrontamiento con el nivel educativo o con el nivel económico, han observado que las personas mayores con un nivel educativo más alto, es más probable que utilicen el afrontamiento dirigido al problema y menos el dirigido a la emoción. Estas personas racionalizan más sus elecciones y no son tan rígidas; además, son capaces de obtener recursos económicos que les permitan manejar sus problemas directamente. Por el contrario, las personas mayores con niveles educativos bajos confían más en sus creencias religiosas y controlan en mayor medida sus sentimientos y emociones (Martin, Rott, Poon, Courtenay y Lehr, 2000).

De este modo y dada la importancia de las estrategias de afrontamiento en la última etapa del ciclo vital, y de cómo ciertas variables sociodemográficas pueden modificar la aplicación de éstas, se pretende analizar si existe algún tipo de relación entre la edad y el tipo de estrategias de afrontamiento puestas en marcha, así como analizar la existencia de diferencias en el uso de las estrategias de afrontamiento en función del género, el estado civil, el nivel académico y el nivel de ingresos.

\section{Participantes}

\section{Método}

La muestra utilizada para el desarrollo de esta investigación es de carácter incidental compuesta por 275 adultos mayores que han participado de forma voluntaria y que acuden a un centro de mayores para realizar actividades, con edades comprendidas entre 65 y 95 años, con una media de edad de $73,69(D T=6,24)$, de los cuales el $64,4 \%$ mujeres. Por lo que respecta al estado civil un $56,4 \%$ son casados, $7,6 \%$ solteros y $36 \%$ viudos. En cuanto al nivel de estudios, 21,8\% sabe leer 
y escribir, un $56 \%$ tiene primarios, $13,8 \%$ secundarios y $8,4 \%$ universitarios. Finalmente en relación al nivel de ingresos el $38,5 \%$ percibían ingresos inferiores a 633,80 dólares, un $32,7 \%$ entre 633,80 y 1056,33 dólares, $21,2 \%$ entre 1057,39 y 1584,50 dólares , y el 7,6\% superiores a 1584,50 dólares. Todos dieron su consentimiento a la hora de participar en el estudio. Como criterio de exclusión se utilizó en MMSE (Lobo, Saz, y Marcos, 2002) aquellas personas con una puntuación inferior a 24 fueron excluidos del estudio.

\section{Instrumentos}

Para la realización del estudio se obtuvieron una serie de datos sociodemográficos, género, edad, estado civil, nivel de estudios y finalmente el nivel de ingresos. Además, para evaluar las estrategias de afrontamiento los sujetos respondieron al Cuestionario de Afrontamiento del Estrés (CAE; Sandín y Chorot, 2003). El CAE, es una medida de afrontamiento generalizado de autoinforme de 42 ítems con respuesta tipo Likert graduada entre "nunca" (0) y "casi siempre2 (4). Este cuestionario ha sido desarrollado y validado en España, y a partir de estudios exploratorios y confirmatorios (Tomás, Sancho y Meléndez, 2013) ha mostrado una adecuada estructura factorial obteniéndose siete dimensiones que se presentan a continuación, incluyendo alguno de los ítems que lo componen: 1. Focalizado en la solución del problema, estrategias cognitivas y conductuales encaminadas al estrés, modificando la situación que lo produce ("Pensar detenidamente los pasos a seguir para enfrentarme al problema"); 2. Autofocalización negativa, estrategias basadas en la autoinculpación y la autocrítica por la ocurrencia de la situación estresante o su manejo inadecuado ("Me sentí indefenso/a e incapaz de hacer algo positivo para cambiar la situación”); 3. Reevaluación positiva, estrategias cognitivas que modifican el significado de la situación estresante ("Intenté sacar algo positivo del problema"); 4. Expresión emocional abierta, estrategias encaminadas a liberar emociones que acontecen el proceso de estrés ("Descargué mi mal humor con los demás”); 5. Evitación, estrategias que incluyen la negación y evitación de pensamiento o conductas relacionadas con el acontecimiento estresante ("Cuando me venía a la cabeza el problema, trataba de concentrarme en otras cosas"); 6. Búsqueda de apoyo social, apoyo en otros para modificar la situación estresante o sentir algo de consuelo ("Hablé con amigos o familiares para que me tranquilizaran cuando me encontraba mal"); 7. Religión, recurrir a pensamientos religiosos, a la iglesia, a Dios para modificar la situación estresante o sentir algo de consuelo ("Acudí a la iglesia para rogar que se solucionase el problema”).

El afrontamiento centrado en el problema, está compuesto por las dimensiones focalizado en la solución de problemas, reevaluación positiva y búsqueda de apoyo social; y el centrado en la emoción, por autofocalización negativa, expresión emocional abierta, evitación y religión. Cabe señalar que la literatura (Folkman y Lazarus, 1980) ha indicado que la búsqueda de apoyo social puede saturar de forma positiva en los dos factores, no obstante, en los resultados obtenidos en la escala original (Sandín y Chorot, 2003) exclusivamente saturó en centradas en el problema.

Con respecto a la coherencia interna del cuestionario, se analizó el alfa de Cronbach para cada uno de los siete factores propuestos, obteniendo los siguientes coeficientes: focalizado en solución de problemas $(\alpha=, 77)$, autofocalización negativa $(\alpha=0,68)$, reevaluación positiva $(\alpha=, 66)$, expresión emocional abierta $(\alpha=, 68)$, evitación $(\alpha=, 66)$, búsqueda de apoyo social $(\alpha=, 86)$, y religión $(\alpha=, 93)$.

\section{Análisis de datos}

Los análisis estadísticos se realizaron en el paquete estadístico SPSS (versión 19), aplicándose correlaciones de Pearson para analizar la relación de las medidas con la edad; para el análisis de las medias, se han utilizado pruebas $t$ para muestras independientes y análisis de varianza (ANOVAs) con sus correspondientes pruebas post hoc.

\section{Resultados}

En primer lugar se estudió, si existían relaciones significativas de la edad con cada una de las estrategias de afrontamiento, obteniéndose tan sólo una correlación significativa y positiva con la dimensión religión $(r=, 157 ; p=$ ,001).

A continuación, se estudió la normalidad y la homogeneidad de las varianzas, asumiendo varianzas iguales ya que la significación de Levene era superior a ,05.

Posteriormente, se analizó la posible existencia de diferencias en las estrategias de afrontamiento en función del género, cuyas medias y desviaciones típicas se pueden observar en la tabla 1, obteniéndose resultados significativos en las dimensiones de evitación $(t(273)=2,49 ; p=, 013)$, búsqueda de apoyo social $(t(273)=2,49 ; p=, 013)$ y religión $(t(273)=5,59$; $p=, 001)$, siendo en todos los casos las mujeres quienes obtienen puntuaciones superiores.

Para el estudio de las diferencias en función del estado civil, se aplicó análisis de varianza, obteniéndose diferencias significativas en reevaluación positiva $(F(2,70)=3,42 ; p=, 034)$, búsqueda de apoyo social $(F(2,70)=3,38 ; p=, 035)$ y religión $(F(2,70)=7,20 ; p=, 001)$. La prueba post hoc de Tukey para el contraste de medias mostró diferencias en la reevaluación positiva entre los grupos de solteros y viudos $(p=, 026)$; entre casados y solteros en búsqueda de apoyo social $(p=, 040)$; y en religión entre casados y viudos $(p=, 001)$.

Por lo que respecta a la variable nivel de estudios, el ANOVA mostró diferencias significativas para dos de las estrategias de afrontamiento, autofocalización negativa $(F(3,266)=3,24$; $p=, 023)$ y evitación $(F(3,266)=3,15 ; p=, 025)$. La prueba post hoc de Tukey mostró en autofocalización negativa diferencias entre sin estudios y estudios universitarios $(p=, 050)$; y en evitación entre sin estudios y secundaria $(p=, 027)$. 
ESTRATEGIAS DE AFRONTAMIENTO EN MAYORES

Tabla 1

Medias y desviación típica de las estrategias de afrontamiento en función de los sociodemográficos.

\begin{tabular}{|c|c|c|c|c|c|c|c|}
\hline & FSP & AFN & REP & EEA & EVT & BAS & RLG \\
\hline & $M(D T)$ & $M(D T)$ & $\begin{array}{l}\text { Género } \\
M(D T)\end{array}$ & $M(D T)$ & $M(D T)$ & $M(D T)$ & $M(D T)$ \\
\hline Hombres & $2,77(, 84)$ & $1,65(, 82)$ & $2,68(, 78)$ & $1,33(, 84)$ & $1,64(, 86)$ & $1,94(1,10)$ & $1,25(1,39)$ \\
\hline \multirow[t]{2}{*}{ Mujeres } & $2,55(, 90)$ & $1,71(, 83)$ & $2,67(, 76)$ & $1,45(, 88)$ & $1,93(, 95)$ & $2,30(1,12)$ & $2,26(1,31)$ \\
\hline & $M(D T)$ & $M(D T)$ & $\begin{array}{c}\text { Estado Civil } \\
M(D T)\end{array}$ & $M(D T)$ & $M(D T)$ & $M(D T)$ & $M(D T)$ \\
\hline Casado & $2,66(, 88)$ & $1,68(, 81)$ & $2,69(, 68)$ & $1,33(, 82)$ & $1,73(, 88)$ & $2,04(1,08)$ & $1,64(1,40)$ \\
\hline Soltero & $2,94(, 83)$ & $1,56(, 88)$ & $3,09(, 62)$ & $1,51(, 82)$ & 2,18 (,98) & $2,63(1,20)$ & $1,76(1,50)$ \\
\hline \multirow[t]{2}{*}{ Viudo } & $2,55(, 87)$ & $1,71(, 85)$ & $2,60(, 88)$ & $1,51(, 94)$ & $1,90(, 99)$ & $2,31(1,14)$ & $2,32(1,36)$ \\
\hline & $M(D T)$ & $M(D T)$ & $\begin{array}{l}\text { Estudios } \\
M(D T)\end{array}$ & $M(D T)$ & $M(D T)$ & $M(D T)$ & $M(D T)$ \\
\hline Sin estudios & $2,54(, 98)$ & $1,93(, 78)$ & $2,76(, 76)$ & $1,51(, 86)$ & $2,10(, 90)$ & $2,33(1,20)$ & $2,20(1,39)$ \\
\hline Básicos & $2,63(, 86)$ & $1,61(, 83)$ & $2,65(, 77)$ & $1,45(, 92)$ & $1,80(, 93)$ & $2,14(1,13)$ & $1,88(1,42)$ \\
\hline Secundaria & $2,66(, 79)$ & $1,79(, 91)$ & $2,65(, 75)$ & $1,17(, 74)$ & $1,54(, 88)$ & $2,05(1,10)$ & $1,76(1,49)$ \\
\hline \multirow[t]{2}{*}{ Universitarios } & $3,09(, 59)$ & $1,41(, 72)$ & $2,80(, 71)$ & $1,26(, 64)$ & $1,65(, 93)$ & $2,30(, 88)$ & $1,46(1,46)$ \\
\hline & $M(D T)$ & $M(D T)$ & $\begin{array}{l}\text { Ingresos } \\
M(D T)\end{array}$ & $M(D T)$ & $M(D T)$ & $M(D T)$ & $M(D T)$ \\
\hline$<633,80 \$$ & $2,59(, 88)$ & $1,80(, 80)$ & $2,60(, 83)$ & $1,39(, 90)$ & $1,88(, 97)$ & $2,32(1,10)$ & $2,17(1,45)$ \\
\hline $633,80-1056,33 \$$ & $2,62(, 83)$ & $1,60(, 78)$ & $2,73(, 71)$ & $1,44(, 84)$ & $1,79(, 91)$ & $2,12(1,15)$ & $1,98(1,37)$ \\
\hline $1057,39-1584,50 \$$ & $2,84(, 86)$ & $1,65(, 90)$ & $2,74(, 70)$ & $1,31(, 93)$ & $1,72(, 97)$ & $2,05(1,23)$ & $1,09(1,22)$ \\
\hline$>1584,50 \$$ & $3,13(, 58)$ & $1,44(, 91)$ & $2,89(, 81)$ & $1,35(, 64)$ & $1,80(, 93)$ & $2,10(, 94)$ & $1,37(1,45)$ \\
\hline
\end{tabular}

Nota: $\mathrm{FSP}=$ Focalizado solución problema; $\mathrm{AFN}=$ Autofocalización negativa; $\mathrm{REP}=$ Reevaluación positiva; EEA=Expresión emocional abierta; EVT=Evitación; BAS=Búsqueda de apoyo social; $R L G=$ Religión.

Finalmente el ANOVA para la variable nivel de ingresos mostró diferencias en las estrategias de focalizado en solución de problemas $(F(3,252)=2,99 ; p=, 031)$ y religión $(F(3,252)=6,83$; $p=, 001)$. La prueba post hoc mostró diferencias entre los de más de 1584,50 dólares y los de menos de 633,80 dólares para la estrategia focalizado en solución de problemas $(p=, 040)$; en religión se observaron diferencias entre el grupo de 1057,391584,50 dólares y los de menos de 633,80 dólares $(p<, 001)$ y los de 633,80 y 1056,33 dólares $(p=, 006)$.

\section{Discusión}

El envejecimiento es una etapa en la que se producen una serie de pérdidas a las cuales la persona mayor debe adaptarse y así poder mantener su bienestar.

Una importante variable mediadora es el afrontamiento, que a través de sus diferentes estrategias, permite adaptarse a las situaciones dadas en esta etapa del ciclo vital, es decir, la puesta en marcha de los propios recursos para la consecución de objetivos y la manipulación de la situación para conseguir un buen ajuste. En esta última etapa, existe una tendencia evolutiva hacia un tipo de estrategias adaptativas sustentadas en mayor medida en la acomodación a las nuevas situaciones y no tanto en la asimilación (Meléndez, Tomás y Navarro, 2008). De este modo, los mayores tienden, bien a reajustar sus metas manteniendo tanto lo ya conseguido como lo disponible y posible, o bien a sustituir sus objetivos por otros más realistas, lo cual ayuda a optimizar los recursos y a tener un mayor control de la situación. Es de señalar la importancia que tiene el afrontamiento para el bienestar de las personas mayores tal y como señalan Tomás, Sancho, Melendez, y Mayordomo (2012) mediante un modelo de ecuaciones estructurales que confirma la predicción del bienestar a partir del afrontamiento.
En cuanto a los resultados obtenidos con respecto a la relación entre las estrategias de afrontamiento y la edad, parecen ser contradictorios a los encontrados en la literatura (Carver y Connor-Smith, 2010; Trouillet et al., 2009), ya que la edad no muestra relación con las estrategias de afrontamiento, sino que permanece estable a lo largo del tiempo. Por otro lado, un estudio longitudinal demostró que el uso de ambos tipos de afrontamiento disminuyen con la edad, excepto para el uso de la búsqueda de apoyo social, que se mantiene estable en el tiempo (Brennan, Holanda, Schutte y Moos, 2012).A excepción de la estrategia religión, que mostró una relación significativa y positiva con la edad, el resto de las estrategias no mostraron relación significativa. Cabe señalar que el uso de este tipo de estrategias en personas mayores ha sido demostrado como una característica diferencial en comparación con jóvenes y adultos (Meléndez, Mayordomo, Sancho y Tomás, 2012). Además, tal y como se señalaba, esta es una etapa con importantes pérdidas sobre las que el sujeto mayor no dispone de control, de modo que la aplicación de este tipo de estrategias puede facilitar la adaptación. Ésta parece jugar un importante papel en el afrontamiento del estrés y en la conservación de la salud en la adultez tardía o vejez (RiveraLedesma y Montero-López, 2005), influyendo positivamente en la salud y en el sentido de bienestar personal (Clark et al., 1996), y relacionándose inversamente con depresión (Koenig et al., 1992) y soledad (Walton, Shultz, Beck y Walls, 1991); además, autores como Bosworth, Park, McQuoid, Hays y Steffens (2003) indican que los mecanismos religiosos pueden ser positivos para la reevaluación que se da ante una situación estresante.

Por lo que respecta al género, se ha observado cómo las mujeres utilizan más que los hombres las estrategias centradas en 
la emoción, resultados coherentes con otros trabajos (Meléndez, Mayordomo, Sancho y Tomás, 2012; Rose y Rudolph, 2006; Tamres, Janicki y Helgeson, 2002). Los resultados de este trabajo han mostrado diferencias en evitación, búsqueda de apoyo social y religión, resultados similares a los obtenidos por Navarro y Bueno (2005) en un estudio realizado con muestra de adultos muy mayores, en el que las mujeres utilizan más la búsqueda de apoyo social y la evitación. A modo de explicación de los hallazgos encontrados, cabe señalar que este tipo de diferencias pueden ser consistentemente contrastadas bajo la hipótesis de la socialización, que predice que los hombres son instruidos para un uso más activo e instrumental en las conductas de afrontamiento, mientras que a las mujeres se les enseña a utilizar comportamientos más pasivos y centrados en las emociones (Mataud, 2004). Otros autores (Yeh, Huang, Chou y Wan, 2009) señalan que los dos géneros operan en diferentes contextos sociales, y por lo tanto tienden a desarrollar diferentes respuestas; estando definidos los roles femeninos por su capacidad para experimentar, expresar y comunicar su emociones a otras personas, y también para empatizar con los sentimientos de los demás; mientras que el rol masculino está definido por la propia capacidad para reprimir y controlar sus emociones.

El estado civil mostró diferencias en búsqueda de apoyo social, reevaluación positiva y religión. De forma más concreta, los resultados señalaron que en búsqueda de apoyo social los solteros aplican más esta estrategia, posiblemente debido a la existencia una mayor estructura de red social, es de señalar que el uso de esta estrategia repercute positivamente tanto en la salud como en el bienestar (Antonucci, 2001); en religión, se vio que los viudos utilizaban más esta estrategia que los casados, pudiendo poner en relación el mayor uso de esta estrategia por parte de los viudos con la autoeficacia percibida (Schwarzer, 2001), ya que están enfrentándose a una situación sobre la que no tienen ningún control; teniendo en cuenta esta disminución en la autoeficacia percibida, también podría señalarse el hecho de que los viudos utilicen en menor medida que los solteros la reevaluación positiva de la situación como estrategia adaptativa, ya que no perciben ningún control sobre la situación a la que se están enfrentando (la viudedad) y por lo tanto no realizan evaluaciones nuevas de la misma, lo que podría dificultar la positivización de una situación dañina o desadaptativa en esta etapa del ciclo vital.

De cara al nivel de estudios, se obtuvieron diferencias significativas en las estrategias autofocalización negativa y evitación, siendo el grupo de sin estudios, en ambas estrategias el que obtiene puntuaciones más altas, es decir, las personas con estudios inferiores o sin estudios hacen mayor uso de la autoinculpación y negación de pensamiento o de las conductas relacionadas con el acontecimiento estresante. Estos resultados son similares a los encontrados por Martin et al. (2000) quienes señalan que los mayores con menos estudios hacen mayor uso de las estrategias centradas en la emoción que los que tienen estudios superiores que se enfrentan de un modo más directo a la situación estresante. Desde una perspectiva cognitiva del envejecimiento, una posible explicación a la utilización de recursos centrados en el problema sería el poseer una mayor capacidad de reserva cognitiva. Tal y como indica Stern (2009), a mayor nivel de estudios mayor capacidad de reserva cognitiva, lo que les hace plantearse mayor número de posibilidades o hacer visibles aquellos recursos propios para enfrentarse a una situación desadaptativa.

En cuanto a la última variable sociodemográfica estudiada, el nivel de ingresos, los resultados mostraron que existen diferencias en las estrategias focalizado en solución de problemas y religión. Las personas con mayor nivel económico utilizan en mayor medida la estrategia focalizado en solución de problemas que las personas con ingresos inferiores; por el contrario, las personas con menor nivel de ingresos, hacen un mayor uso de la estrategia religión que las personas con ingresos superiores. En esta misma línea de resultados, Navarro y Bueno (2005) señalaron que los adultos muy mayores con un nivel económico medio-alto, aceptan más las consecuencias derivadas de sus problemas, aplazan más cualquier tipo de afrontamiento hasta obtener mayor información y tienden a suprimir cognitivamente los efectos emocionales generados por la situación.

Finalmente, cabe señalar que es importante el estudio de las estrategias de afrontamiento utilizadas por los adultos mayores dado que estas ofrecen información en relación a cómo los mayores se enfrentan a determinadas situaciones para obtener resultados más adaptativos. Por otra parte, este trabajo presenta una serie de limitaciones, ya que el tipo de muestreo es incidental, y además, la posible existencia de un efecto de cohorte puede repercutir en los resultados obtenidos, ya que puede estar muy influenciada por características normativas de carácter socio-histórico. Si bien, este trabajo plantea nuevas perspectivas de trabajo e investigación, como la puesta en marcha de posibles talleres o sesiones de formación que ayuden a desarrollar estrategias adaptativas y fomentar de modo indirecto el bienestar psicológico y la satisfacción vital, mejorando por tanto el proceso adaptativo del sujeto mayor; así como el estudio de la autoeficacia percibida y su papel con las estrategias de afrontamiento y por tanto con el bienestar y la adaptación en el envejecimiento.

\section{Referencias}

1. Antonucci, T. C. (2001). Social relations: An examination of social networks, social support and sense of control. En J. E. Birren y E. Schaie (Eds.), Handbook of the psychology of aging (pp. 427-453). New York: Academic Press.

2. Brennan, P., Holland, J., Schutte, K. y Moos, R. (2012). Coping trajectories in later life: A 20-year predictive study. Aging and Mental Health, 16, 305-316. http://dx.doi.org/1 $\underline{0.1080 / 13607863.2011 .628975}$

3. Birren, J. E. (1994). Consumer decision making and age: maintaining resources and independence. Philadelphia: University of Pennsylvania. 
4. Bosworth, H. B., Park, K.S., McQuoid, D.R., Hays, J. C. y Steffens, D.C. (2003). The impact of religious practice and religious coping on geriatric depression. International Journal of Geriatric Psychiatry, 18, 905-914. http://dx.doi. org/10.1002/gps.945

5. Carver, C. S. y Connor-Smith, J. (2010). Personality and coping. Annual Review of Psychology, 61, 679-704 http:// dx.doi.org/10.1146/annurev.psych.093008.100352

6. Clark, F., Carlson, M., Zemke, R., Frank, G., Patterson, K., Ennevor, B. L., ... y Lipson, L. (1996). Life domains and adaptive strategies of a group of low-income, well older adults. American Journal of Occupational Therapy, 50, 99108. http://dx.doi.org/10.5014/ajot.50.2.99

7. Folkman, S. y Lazarus, R. S. (1980). An analysis of coping in a middle-aged community sample. Journal of Health and Social Behavior, 21, 219-239. http://dx.doi. org $/ 10.2307 / 2136617$

8. Folkman, S., Lazarus, R. S., Pimley, S. y Novacek, J. (1987). Age differences in stress and coping processes. Psychology and Aging, 2, 171-184. http://dx.doi.org/10.1037/0882$\underline{7974.2 .2 .171}$

9. Freund, A. M. y Riediger, M. (2001). What I have and what I do-The role of resource loss and gain throughout life. Applied Psychology, 50, 370-380.

10. Holahan, C. J. y Moos, R. H. (1987). Personal and contextual determinants of coping strategies. Journal of Personality and Social Psychology, 52, 946-955. http:// dx.doi.org/10.1037/0022-3514.52.5.946

11. Kirchner, T., Forns, M., Muñoz, D. y Pereda, N. (2008). Psychometric properties and dimensional structure of the Spanish version of the Coping Responses Inventory-Adult Form. Psicothema, 20, 902-909.

12. Koenig, H. G., Cohen, H. J., Blazer, D. G., Pieper, C., Meador, K. G., Shelp, F., ... y DiPasquale, B. (1992). Religious coping and depression among elderly, hospitalized medically ill men. The American Journal of Psychiatry, 149, 1693-1700. http://dx.doi.org/10.1176/ajp.149.12.1693

13. Labouvie-Vief G. (1999). Emotions in adulthood. In V. L. Bengtson \& K.W. Schaie (Eds.), Handbook of theories of aging (pp. 253-267). New York: Springer.

14. Lazarus, R. S. y Folkman, S. (1984). Stress appraisal and coping. New York: Springer.

15. Lobo A, Saz P, Marcos G. 2002. Adaptación del Examen Cognoscitivo Mini-Metal. [Adaptation of Mini-Mental State Examination]. Madrid: Tea Ediciones.

16. Martin, P., Kliegel, M., Rott, C., Poon, L. W. y Johnson, M. A. (2008). Age differences and changes of coping behavior in three age groups: Findings from the Georgia Centenarian Study. The International Journal of Aging and Human Development, 66, 97-114. $\quad$ http://dx.doi.org/10.2190/ AG.66.2.a

17. Martin, P., Rott, C., Poon, L. W., Courtenay, B. y Lehr, U. (2000). A molecular view of coping behaviour in older adults. Journal of Aging and Health,13, 72-79. http:// dx.doi.org/10.1177/089826430101300104

18. Matud, M. P. (2004). Gender differences in stress and coping styles. Personality and Individual Differences, 37, 1401-1415. http://dx.doi.org/10.1016/j.paid.2004.01.010

19. Meléndez, J. C., Mayordomo, T., Sancho, P. y Tomás, J. M. (2012). Coping strategies: Gender differences and development throughout life span. The Spanish Journal of Psychology, 15, 1089-1098. http://dx.doi.org/10.5209/ rev_SJOP.2012.v15.n3.39399

20. Meléndez, J. C., Tomás, J. M. y Navarro, E. (2008). Análisis del bienestar en la vejez según la edad. Revista Española de Geriatría y Gerontología, 43, 90-95. http:// dx.doi.org/10.1016/S0211-139X(08)71161-2

21. Navarro, A. B. y Bueno, B. (2005). Efectos del sexo, el nivel educativo y el nivel económico en el afrontamiento de los problemas en personas muy mayores. Revista Española de Geriatría y Gerontología, 40, 34-43. http://dx.doi. org/10.1016/S0211-139X(05)74820-4

22. Rivera-Ledesma, A. y Montero, M. (2005). Espiritualidad y religiosidad en adultos mayores mexicanos. Salud Mental, $28,51-58$.

23. Rose, A. J. y Rudolph, K. D. (2006). A review of sex differences in peer relationship processes: potential tradeoffs for the emotional and behavioral development of girls and boys. Psychological Bulletin, 132, 98-131. http:// dx.doi.org/10.1037/0033-2909.132.1.98

24. Sandín, B. y Chorot, P. (2003). Cuestionario de Afrontamiento del Estrés (CAE): Desarrollo y validación preliminar. Revista de Psicopatología y Psicología Clínica, 8, 39-54. http://dx.doi.org/10.5944/rppc. vol.8.num.1.2003.3941

25. Schwarzer, R. (2001). Social-cognitive factors in changing health-related behaviors. Current Directions in Psychological Science, 10, 47-51. http://dx.doi. org/10.1111/1467-8721.00112

26. Stern, Y. (2009). Cognitive reserve. Neuropsychologia, 47, 2015-2028. http://dx.doi.org/10.1016/j.neuropsychologia.2 $\underline{009.03 .004}$

27. Tamres, L. K., Janicki, D. y Helgeson, V. S. (2002). Sex differences in coping behavior: A meta-analytic review and an examination of relative coping. Personality and Social Psychology Review, 6, 2-30. http://dx.doi.org/10.1207/ S15327957PSPR0601 1

28. Taylor, S. E., y Stanton, A. (2007). Coping resources, coping processes, and mental health. Annual Review of Clinical Psychology, 3, 129-153. http://dx.doi.org/10.1146/ annurev.clinpsy.3.022806.091520

29. Tomás, J. M., Sancho, P., Melendez, J. C. y Mayordomo, T. (2012). Resilience and coping as predictors of general well-being in the elderly: A structural equation modeling approach. Aging y Mental Health, 16, 317-326. http:// dx.doi.org/10.1080/13607863.2011.615737 
30. Tomás, J. M., Sancho, P. y Meléndez, J. C. (2013). Validación del Cuestionario de afrontamiento del estrés (CAE) para su uso en población mayor española. Behavioral Psychology/ Psicologia Conductual, 21, 103-122.

31. Trouillet , R. , Gana , K. , Lourel , M. y Fort , I. ( 2009 ). Predictive value of age for coping: The role of selfefficacy, social support satisfaction and perceived stress. Aging and Mental Health, 13, 357-366. http://dx.doi. org/10.1080/13607860802626223

32. Walton, C. G., Shultz, C. M., Beck, C. M. y Walls, R. C. (1991). Psychological correlates of loneliness in the older adult. Archives of Psychiatric Nursing, 5, 165-170. http:// dx.doi.org/10.1016/0883-9417(91)90017-Y
33. Yeh, S. J., Huang, C., Chou, H. y Wan, T. T. H. (2009). Gender differences in stress and coping among elderly patients on hemodialysis. Sex Roles, 60, 44-56. http:// dx.doi.org/10.1007/s11199-008-9515-2

Fecha de recepción: 1 de octubre, 2014

Fecha de recepción de la versión modificada: 28 de abril, 2015

Fecha de aceptación: 29 de abril, 2015 